\title{
Field Emission as a tool for Exploring New Phenemena in Nanomechanics
}

\author{
P. Vincent, A. Ayari, S. Perisanu, P. Poncharal, T. Barois, A. Derouet, M. Choueib, and
}

\section{$\underline{\text { S.T. Purcell }}$}

Institute Lumière Matière, UMR 5306 CNRS, Université de Lyon 1, Villeurbanne, France

Over these past years our team has been pioneering an original orientation which is the use of field emission (FE) as a probe of mechanically resonating nanotubes (NTs), nanowires (NWs) and recently graphene. The resonators and electrical environment can be considered to be a nano-electro-mechanical system (NEMS). My goal here is to promote this subject better within the FE community whereas it has mostly been exposed within the NEMs community.

The principle of FE detection of resonators is simply that the field amplification factor, and hence the field emission current, varies as the resonator apex moves. NEMS applications are still mostly in their fundamental research phase. Though even more true for FE detection because of stricter vacuum requirements, FE NEMS have proved to be an excellent test bed for discovering new concepts and building electro-mechanical models of interest for the wider NEMs community.

After a quick review of NEMS principles, I will set the basis of the rest of the talk by rapidly going over our experimental geometry and samples and initial experiments on NTs. These revealed the FE Microscopy imaging of the resonances, high electrical tuning of the resonance frequency, mechanical response curves, frequency splitting of the two polarisation modes and treating nanowires to have high quality factors.

In the rest of the talk more complex subjects will be evoked such as electrostatic damping of nano-resonators under field, several nonlinear mechanical effects (Duffing response, mode coupling), the FE Nanoradio, and mass sensing with FE atomic resolution. Extra time will be given to the subject of auto-oscillations of FE NEMS which involves concepts such as frequency stability and synchronisation.

If time permits I will present our more recent results on a new concept which is self oscillating phase in auto-oscillators. 